PAPER

\title{
Perceptual characteristics of broken and drop tones in Vietnamese
}

\author{
Tu Trong Do* and Tomio Takara ${ }^{\dagger}$ \\ Department of Information Engineering, University of the Ryukyus, \\ 1 Senbaru, Nishihara, 903-0213 Japan
}

(Received 2 March 2010, Accepted for publication 27 July 2010)

\begin{abstract}
Perceptual characteristics of broken and drop tones in the Vietnamese language are studied. A broken tone syllable has a glottal constriction at the middle part, while a drop tone syllable has a glottal stop at the end and has a short length. In this study, four experiments were conducted. First, expanding from our previous research, the power control for the broken tone was evaluated using several power patterns and its effectiveness was reconfirmed. The power dip pattern makes the synthetic sound of the broken tone highly intelligible. Second, the similarity between the drop tone and the first half of the broken tone were studied. Experimental results showed that they are comparable, i.e., the first half of the broken tone can be replaced by the drop tone, and the first half of the broken tone can be perceived as a drop tone. In the third and fourth experiments, the final parts of drop and broken tones were examined using different fundamental frequency $\left(F_{0}\right)$ patterns. As a result, we found that the drop tone has a perceptual threshold with an $F_{0}$ slope, while $F_{0}$ of the final part of the broken tone has a minor effect on its perception.
\end{abstract}

Keywords: Vietnamese tone, Perception, Glottal feature, Fundamental frequency, Power control

PACS number: 43.70.Mn, 43.71.Gv [doi:10.1250/ast.32.8]

\section{INTRODUCTION}

Vietnamese, the official language of Vietnam, is a tonal language with six lexical tones: level, falling, broken, curve, rising, and drop. Tone is obligatory for a syllable. Different tones change the meanings of syllables that consist of the same phoneme sequence. The six Vietnamese tones with the syllable "ma" are shown in Table 1. Among the six tones, broken and drop tones have glottal features $[1,2]$. The glottal constrictions cause irregular changes around the middle part of the vowel of the broken tone syllable and at the end of the drop tone syllable and shorten the length of the drop tone syllable. Examples of the waveforms, spectrograms, and $F_{0}$ patterns of the syllable "ma" with three tones (level, broken, and drop) are shown in Fig. 1.

Conventionally, tone has been viewed as a distinctive pitch level of a syllable from two aspects: static (high, mid, or low) and dynamic (contour) [3-5]. The fundamental frequency $\left(F_{0}\right)$ has been studied for tone synthesis in several tonal languages such as Chinese, Thai, and Vietnamese. However, using only $F_{0}$ is not sufficient for

*e-mail: dss@iip.ie.u-ryukyu.ac.jp

†e-mail: takara@ie.u-ryukyu.ac.jp the speech production of Vietnamese tone [6]. Both $F_{0}$ and the power patterns are used to synthesize Vietnamese speech effectively [7]. Power control for Vietnamese tones is unique compared with other tonal languages.

In this study, we conducted four experiments. First, the effectiveness of power control was reconfirmed by using several V-shape power patterns. The power dip pattern makes the synthetic sound of the broken tone highly intelligible, and a reasonable weighting factor is 0.875 . Second, we examined the similarity between the drop tone and the first half of the broken tone. The experimental results showed that they are comparable: the drop tone combined with the final part of the broken tone can be perceived as a broken tone, and conversely, the first half of the broken tone can be perceived as a drop tone. In the third and fourth experiments, the final part of the drop tone and that of the broken tone were studied. We found that the perceptual threshold of $F_{0}$ for the drop tone is around $80 \mathrm{~ms}$. For the broken tone perception, the final part has a minor effect.

The organization of this paper is as follows. In Section 2, we describe the common speech material for all experiments. In Section 3, we explain the experiment of power control for the broken tone. In Section 4, we evaluate the similarity of the drop tone and the first half of 
Table 1 The six Vietnamese tones.

\begin{tabular}{lll}
\hline Name (Vietnamese) & Tone mark & \multicolumn{1}{c}{ Example } \\
\hline Level (ngang) & unmarked & ma-ghost \\
Falling (huyen) & grave & mà-that \\
Broken (nga) & tilde & mã-horse \\
Curve (hoi) & hook above & mà-tomb \\
Rising (sac) & acute & má-cheek \\
Drop (nang) & dot below & mạ-rice seedling \\
\hline
\end{tabular}

Table 2 Distinctive features of phonemes in the listening tests.

\begin{tabular}{cl}
\hline Vowel & \multicolumn{1}{c}{ Consonant } \\
\hline /a/ Central, open & /d/ Plosive, alveolar \\
/i/ Front, close & /v/ Fricative, labiodental \\
/u/ Back, close & /m/ Nasal, bilabial \\
\hline
\end{tabular}

the broken tone. In Section 5, we show experiments on the final part of the drop tone. In Section 6, we check the importance of the final part of the broken tone. In Section 7 , we summarize this research.

\section{SPEECH MATERIAL}

We selected vowels and consonants as voiced phonemes that have various distinctive features, as shown in Table 2 [8]. Three vowels /a/, /u/, and /i/ are well known as the vowel triangle. Among the various combinations, three syllables, $/ \mathrm{da} /, / \mathrm{mu} /$, and $/ \mathrm{vi} /$, were used in the evaluations.

The data of six tones were recorded on a digital audio tape (DAT) at a 16-bit sample size and $48 \mathrm{kHz}$ sampling rate, and then down-sampled to $10 \mathrm{kHz}$ on a personal computer. The speech parameters $\left(F_{0}\right.$ with the voiced/ unvoiced information, the power, and the spectrum) were obtained using a short-time cepstral analysis with a time window of $25.6 \mathrm{~ms}$. One frame of the speech signal was $25.6 \mathrm{~ms}$ long and was shifted by $10 \mathrm{~ms}$. For speech synthesis, a source-filter model with a log-magnitude approximation (LMA) filter [9] was implemented. The synthetic sounds were monaural with a $10 \mathrm{kHz}$ sampling rate and 16-bit sample size. The speech was analyzed and synthesized using a visual interactive analysis-synthesis program developed by the authors [10].

Since our purpose was to check the perception of the speech, the evaluations were performed by listening tests. In a test, each sound was played only once. The listeners were instructed to select an answer within two seconds after hearing the sound. The listening tests were carried out inside a sound-proof room. All the sound stimuli were delivered through a Sony MDR-Z900 headphone.
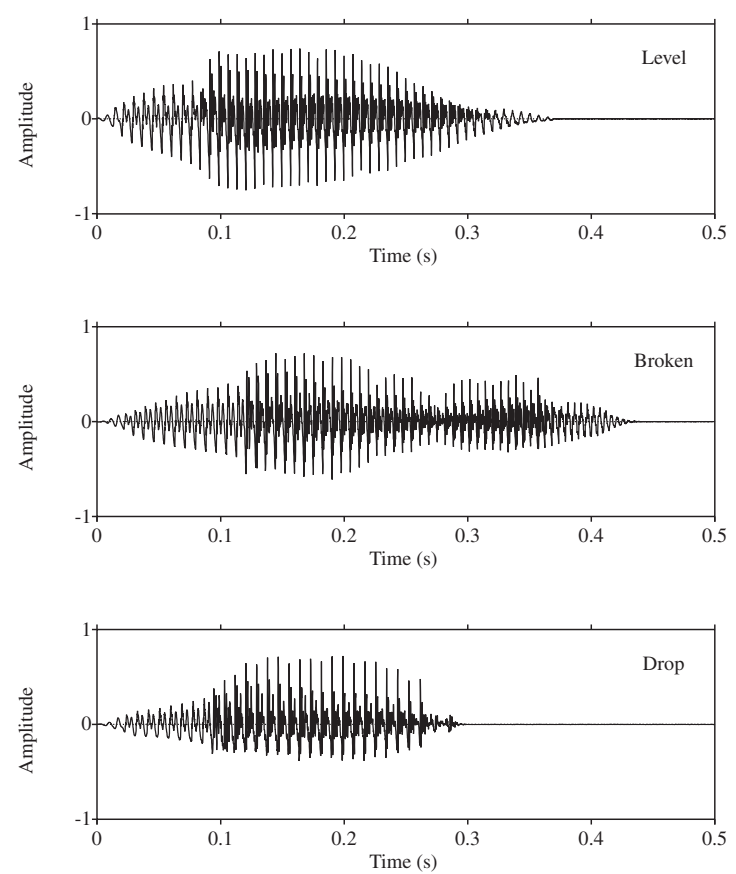

a) Waveform: level, broken, and drop tones
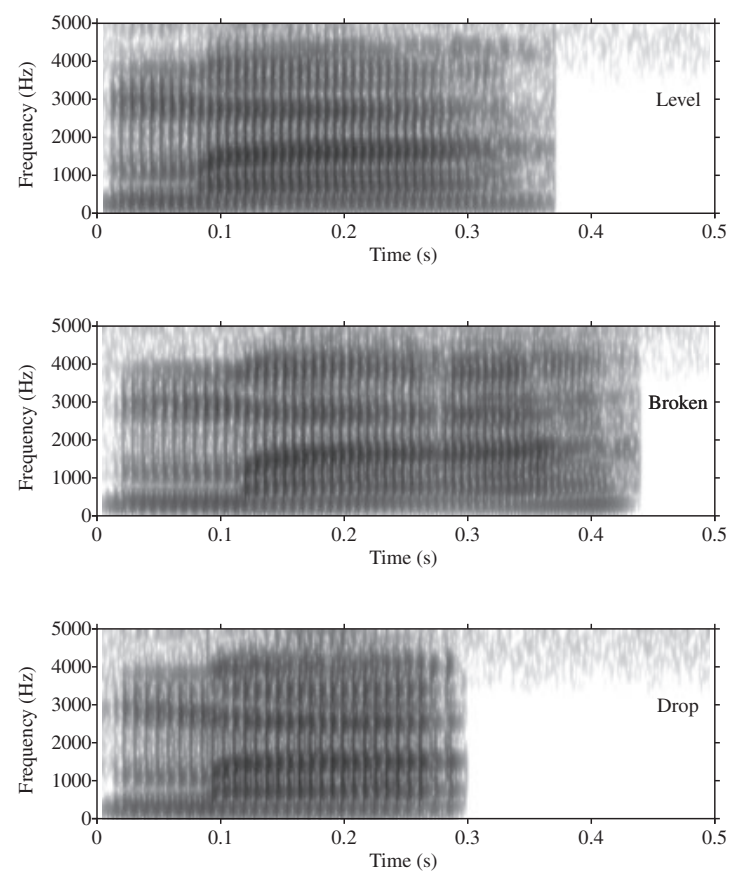

b) Spectrogram: level, broken, and drop tones

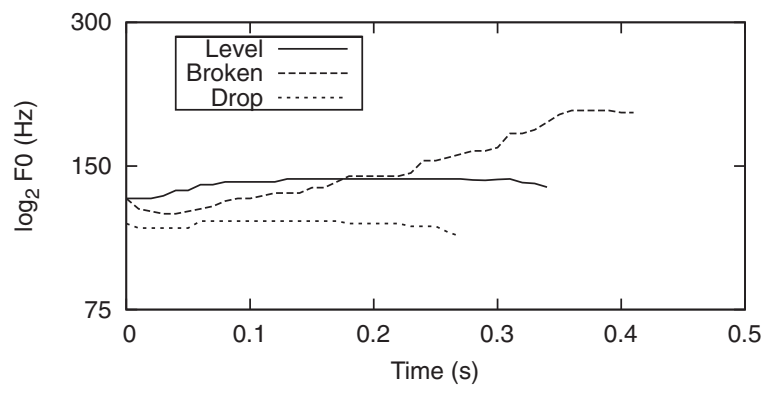

c) Fundamental frequency $\left(F_{0}\right)$ patterns

Fig. 1 Examples of different tones of syllable "ma" uttered by a Vietnamese male. 


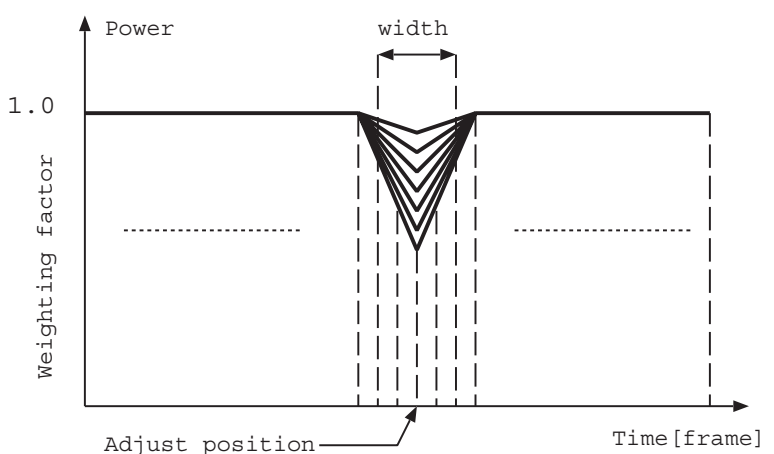

Fig. 2 "Fixed-width" power patterns of broken tone.

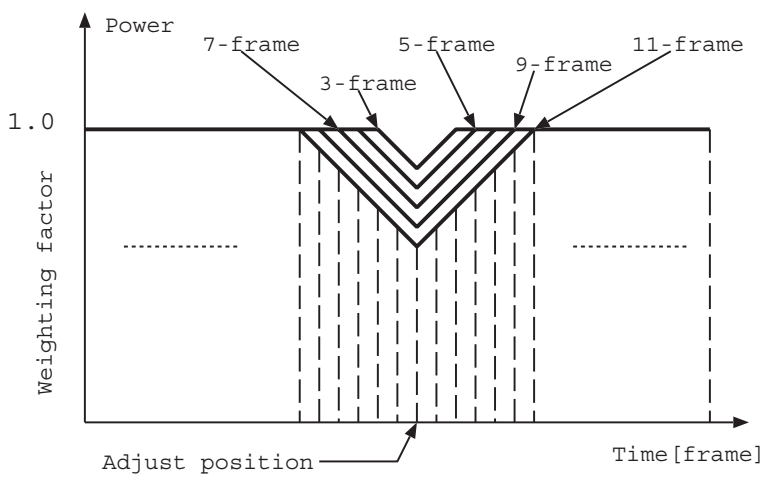

Fig. 3 "Fixed-slope" power patterns of broken tone.

\section{POWER CONTROL FOR BROKEN TONE}

\subsection{Procedure}

To examine the effectiveness of power control, we synthesized the broken tone with the following speech parameters: the average $F_{0}$ of the broken tone [7], the spectrum of level tone, and the power of the level tone with $\mathrm{V}$-shape power control. The adjusted position of the Vshape for each syllable was determined manually around the middle of the vowel with reference to its spectrum. In this experiment, these positions were 18,22 , and 18 frames for /da/, /mu/, and /vi/, respectively. We applied multiple $\mathrm{V}$-shape patterns and evaluated their effect on the intelligibility of synthetic sounds. The width (i.e., how many frames of speech) and the depth (i.e., the power weighting factor) of the V-shape pattern can be changed as follows.

- Change of only the depth (Fig. 2): 5-frame width and seven weighting factors of $0.800,0.850,0.875,0.900$, $0.925,0.950$, and 0.975 . We refer to these changes as "fixed-width" power patterns.

- Change of both the width and the depth (Fig. 3): (3-frame; weighting factor 0.925), (5-frame; weighting factor 0.900), (7-frame; weighting factor 0.875), (9-frame; weighting factor 0.850), (11-frame; weighting factor 0.800), and non-controlled (0-frame; weighting factor 1.000). We refer to these changes as "fixed-slope" power patterns.

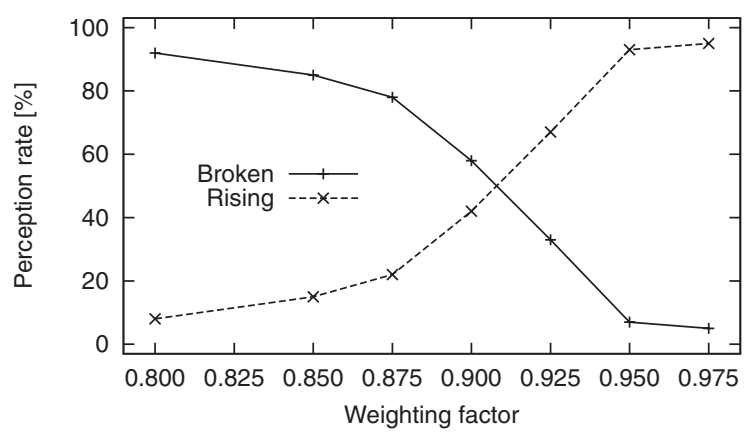

Fig. 4 Perceptions of "fixed-width" patterns.

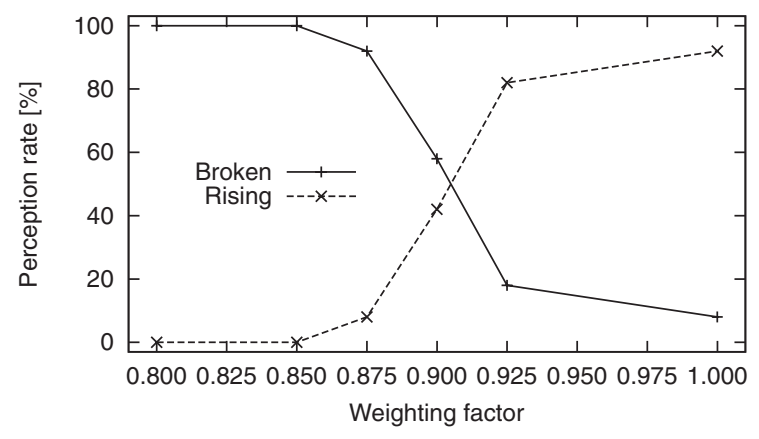

Fig. 5 Perceptions of "fixed-slope" patterns.

A total of 13 patterns of synthetic sounds were combined into the pattern of "/ma/ test-sound $/ \mathrm{ma} /$," wherein /ma/ was the analysis-synthesis sound with the level tone, and "test-sound" was the synthetic sound of /da/, /mu/, or /vi/. The durations of $/ \mathrm{ma} /, / \mathrm{da} /, / \mathrm{mu} /$, and /vi/ were $350,360,420$, and $370 \mathrm{~ms}$, respectively, while the connecting interval was $70 \mathrm{~ms}$. This combination provided a neutral-tone reference to the testing sounds. Each sound was repeated 10 times at random.

In the listening test, the listener selected the identify of the middle syllable of the presented sounds by clicking on one of the six possible Vietnamese syllables on the computer screen. The listeners were two Vietnamese males, Sam and Luong. They were 28 and 26 years old, respectively, and have normal hearing abilities. Both listeners are from northern Vietnam.

In the former study, we evaluated the power control using isolated syllables with the balance of six tones, i.e., the total numbers of syllables with six tones were the same and they were randomly mixed together. The mixing of six tones provided contrasts of the tone among the syllables. In this experiments, separate listening tests were conducted for each syllable, i.e., there was only the broken tone patterns of one syllable in each test.

\subsection{Results}

The averaged results of the listening tests are shown in Figs. 4 and 5. For the "fixed-width" patterns, the perception rate is reasonable with the weighting factor of 0.875 . 
They are higher with smaller weighting factors. We can see that stronger control (i.e., lower power) resulted in a stronger "broken" tone. The greater the weighting factor is, the more rising-like is the tone. The $50 \%$ perception point of the broken-rising tone occurs around the weighting factor of 0.900 . For the "fixed-slope" patterns, a reasonable perception rate is (7-frame; weighting factor 0.875 ), and it becomes higher with smaller weighting factors. The $50 \%$ perception point of broken-rising tones near the $\mathrm{V}$-shape pattern of (5-frame; weighting factor 0.900) can also be observed.

Upon separating listening tests for each syllable, the test conditions become more difficult because we only tested broken tone patterns and did not mix them with other tones. This condition reduces the contrast among synthetic sounds and easily causes confusion between broken and rising tones. It is more precise to check how the listener discriminates the sounds, and the result shows the effectiveness of power control for the broken tone. This experiment reconfirmed that a synthetic broken tone syllable with power control has high intelligibility.

\section{DROP TONE AND FIRST HALF OF BROKEN TONE}

\subsection{Procedure}

Glottal constrictions occur at the middle part of the broken tone syllable and at the end of the drop tone syllable. We created two types of syllables to check if the drop tone and the first half of the broken tone can be exchanged or not.

- Drop tone replaces first half of broken tone. We evaluate how this type is perceived as a broken tone, and refer to this type as "drop-as-half-broken."

- Broken tone cut to retain only the first half. We evaluate how this type is perceived as a drop tone, and refer to this type as "half-broken-as-drop."

Speech parameters of broken and drop tones of three selected syllables $(/ \mathrm{da} /, / \mathrm{mu} /$, and $/ \mathrm{vi} /)$ were obtained. For "drop-as-half-broken," we removed the speech parameters of the first half of the broken tone and inserted those of the drop tone into this part. The replacement was performed without any smoothing at the connection point. For "half-broken-as-drop," we removed the speech parameters of the final part of the broken tone. Since $F_{0}$ of the broken tone was smoothed in the analysis, a rising $F_{0}$ part remained for this type. The $F_{0}$ and power parameters in both types were analysis-synthesis ones. Figure 6 illustrates an example of the waveforms of the syllable "da."

The above two types of synthetic sounds were mixed with the analysis-synthesis sounds of the other five Vietnamese tones in order to balance the tone distribution. In the test, each sound was repeated five times randomly. The listeners responded to the presented sound by selecting

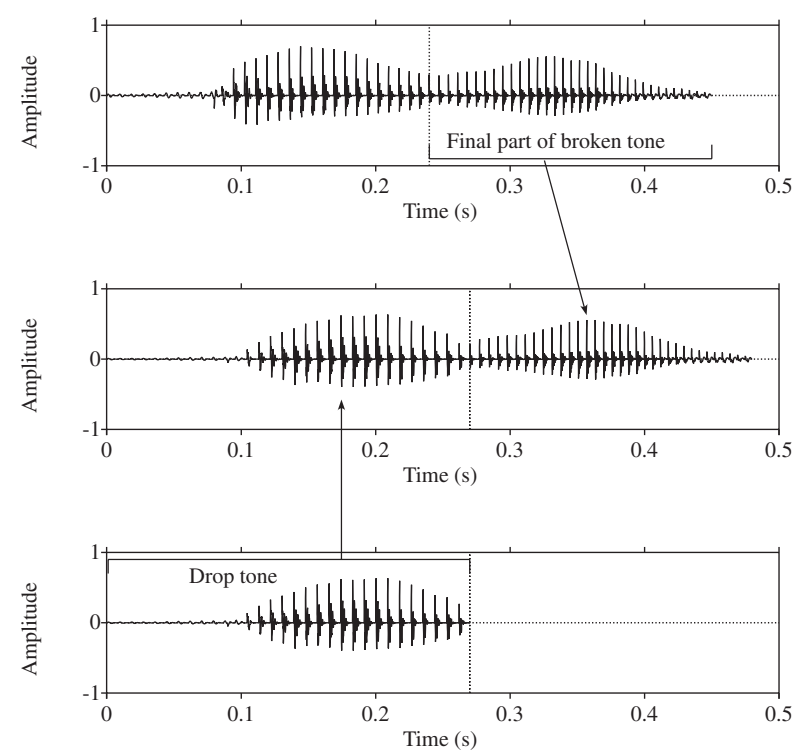

a) Drop-as-half-broken

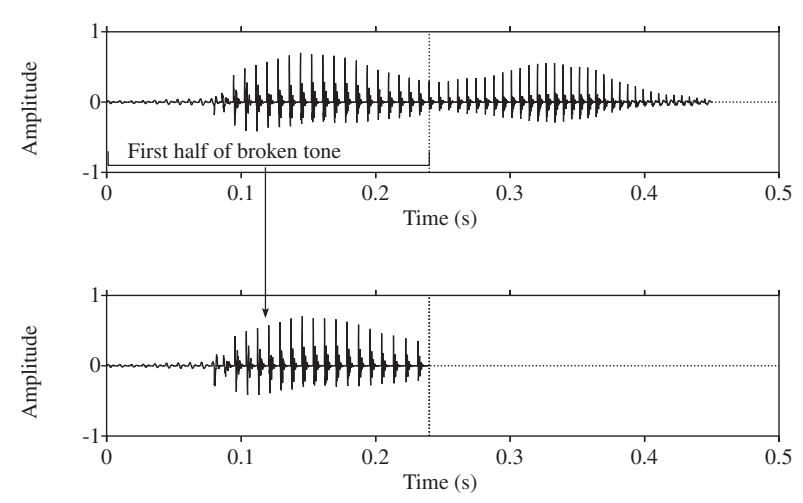

b) Half-broken-as-drop

Fig. 6 Half-broken and drop tones of syllable "da."

one of the six possible Vietnamese syllables. The listeners were Sam and Luong.

\subsection{Results}

For "drop-as-half-broken," the perception rates as the broken tone are $100 \%$ for both Sam and Luong. Clearly, the drop tone can be perceived as the first half of the broken tone. Although there were some distortions when connecting the drop tone to final part of the broken tone directly, they did not affect the intelligibility.

For "half-broken-as-drop," the perception rates as drop are $100 \%$ and $73 \%$ for Luong and Sam, respectively. The error for Sam came from data for $/ \mathrm{mu} /$, in which he could perceive the rising of $F_{0}$ and the sudden stop of the sound. It seemed that such perception followed by silence caused Sam to hear the broken or rising tone. Even with the rising of $F_{0}$ and the sudden stop of the sounds, the perception rates as the drop tone are reasonably high, whereas the length is short. This implies the importance of length in perceiving the drop tone. 
We also performed an extra listening test in which the listener was a female (Phuong) from Southern Vietnam. Despite the dialect differences between Southern and Northern Vietnam, the perception rates for "drop-as-halfbroken" and "half-broken-as-drop" sound types are 100\% and $87 \%$, respectively. This showed that the intelligibility of the synthetic sound is high.

We found that the first half of the broken tone syllable and the drop tone syllable are highly similar and are exchangeable. The difference between these two syllables exists in their final parts. After the relationship between broken and drop tones was shown, we studied the final part of the drop tone.

\section{DROP TONE}

Because of glottal stop at its end, the drop tone has a short length compared with other Vietnamese tones. In this section, experiments to determine the existence of a threshold and the categorical characteristics of the glottal stop of the drop tone are presented.

\subsection{Multislope $\boldsymbol{F}_{\mathbf{0}}$ patterns}

\subsubsection{Intelligibility test}

a) Procedure

In order to synthesize speech, $F_{0}$ contour of the drop tone, the power of the level tone, and the spectrum of the level tone are used. At the end of the syllable, $F_{0}$ was modified with multislope patterns (Fig. 7). To reduce the effect of the power, the average power of the final parts of drop and falling tones was used. There were 10 slopes: 10 , $20,30,40,60,80,100,120,140$, and $160 \mathrm{~ms}$.

The synthetic sounds were combined into a pattern of "/ma/ test-sound /ma/" wherein /ma/ was the analysis-synthesis sound with the level tone and the connecting interval was $70 \mathrm{~ms}$. Since the length is important for the drop tone, we adjusted the test syllable length to the longest slope (i.e., $160 \mathrm{~ms}$ slope) by inserting silence; for example, $10 \mathrm{~ms}$ slope was appended with $150 \mathrm{~ms}$ of silence at the end. The listeners were Sam and Luong.

b) Results

Figure 8 shows the results of the listening test. The synthetic sounds were perceived as drop tones at higher rates when the slopes were smaller. The $80 \mathrm{~ms}$ slope is a reasonable perceptual threshold to discriminate drop from non-drop tones.

Similar experiments were carried out in which we used different power patterns and the average $F_{0}$ pattern of drop and falling tones at the end part. Although we used different power patterns, there were no clear thresholds of changing from drop to non-drop tones. It means $F_{0}$ is more significant than the power in the perception of the drop tone.

There are some languages with glottal features as a phoneme, for example: Danish in Europe and the Ryukyu

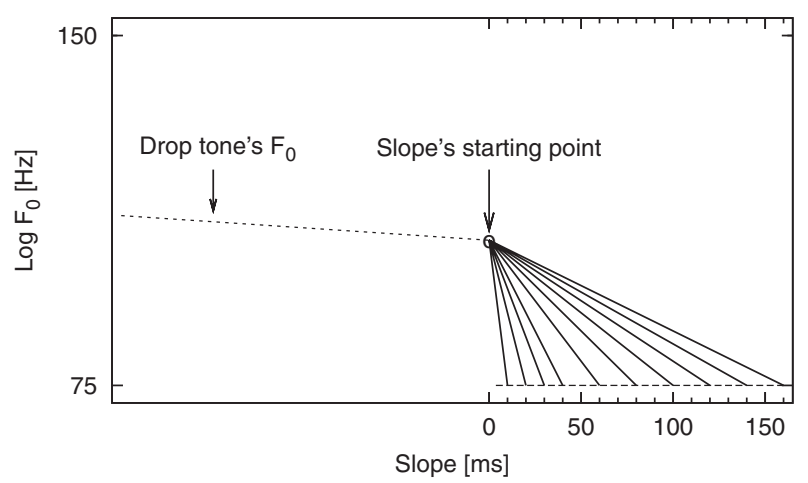

Fig. 7 Multislope $F_{0}$ patterns of drop tone.

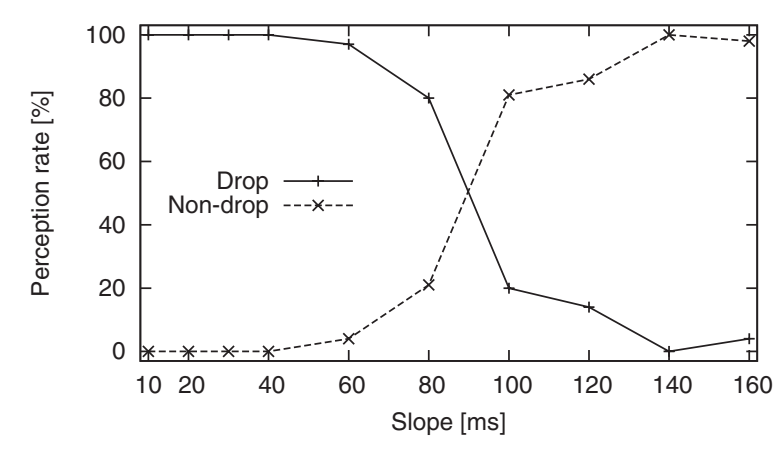

Fig. 8 Perceptions of multislope $F_{0}$ patterns of drop tone.

dialect of Japanese. In the Ryukyu dialect, the glottal explosive was studied for the consonant / $\mathrm{l} /$ that appears at the beginning of the syllable [11]. A similarity between the glottal stop of the drop tone and the glottal explosive $/ \mathrm{P}$ is that $F_{0}$ is the most important parameter when synthesizing the sound and their $F_{0}$ patterns had a threshold. It is interesting that we can see a similarity in the glottal feature in Vietnamese, a tonal language and that in the Ryukyu dialect, a pitch-accent language.

\subsubsection{Categorical test}

a) Procedure

We performed other listening tests to determine whether there is a categorical perception of these multislope patterns or not. A pair of $20 \mathrm{~ms}$ neighbor sounds was played once with a short pause between them. There were 11 pairs and each pair of sounds was repeated 10 times randomly. The listener was asked to decide whether the two sounds are the same tone or not. Each syllable was tested separately. The listeners were Sam and Luong.

b) Results

The results of listening tests are shown in Fig. 9. The peak values of the differences are from "70 90" to "90 110" for Sam and from "90 110" to "110 130" for Luong. For each listener and each sound, there are separate categorical values. In the average result, no consistent category boundary was found. 


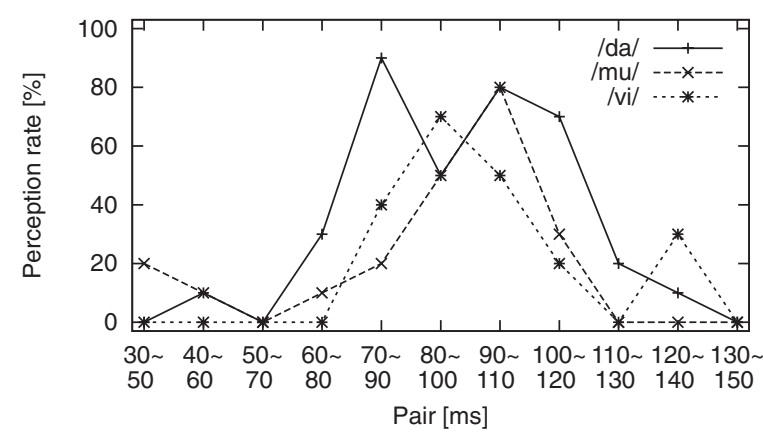

a) Listener: Sam

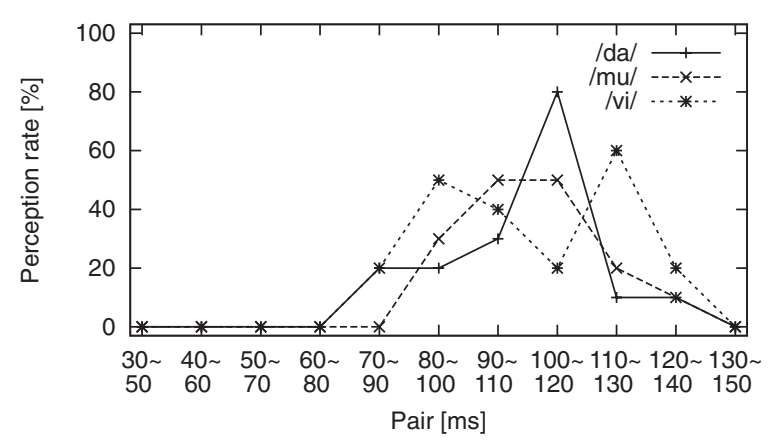

b) Listener: Luong

Fig. 9 Categorical test results for multislope $F_{0}$ patterns of drop tone.

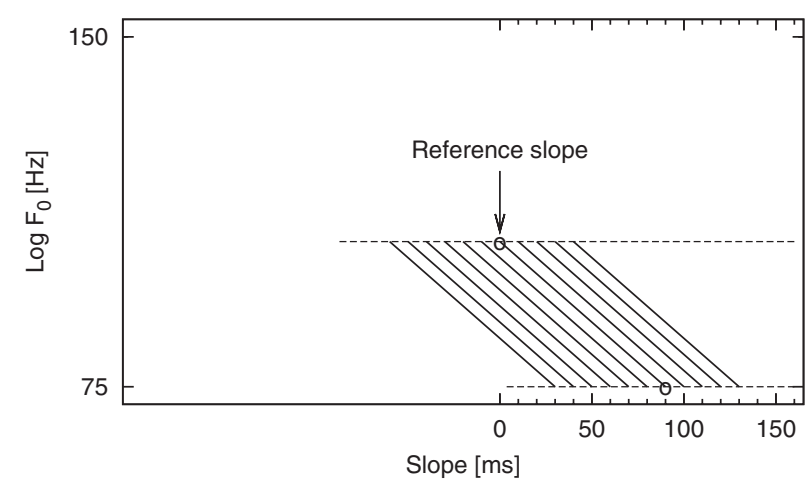

Fig. 10 Shifted $F_{0}$ patterns of drop tone.

\subsection{Shifted $\boldsymbol{F}_{\mathbf{0}}$ patterns}

5.2.1. Intelligibility test

a) Procedure

As seen in Fig. 8, the transition from the drop to nondrop tone is at about $90 \mathrm{~ms}$. We used this timing information as the reference slope to perform further experiments with shift of this slope to the left and right sides. A step of $10 \mathrm{~ms}$ was used to change the length of the drop tone. There were 11 shifted $F_{0}$ patterns (Fig. 10): 30, $40,50,60,70,80,90,100,110,120$, and $130 \mathrm{~ms}$. To synthesize sounds, they were set shorter or longer than the original drop tone. The speech parameters were deleted or expanded at the middle of the vowel with reference to its

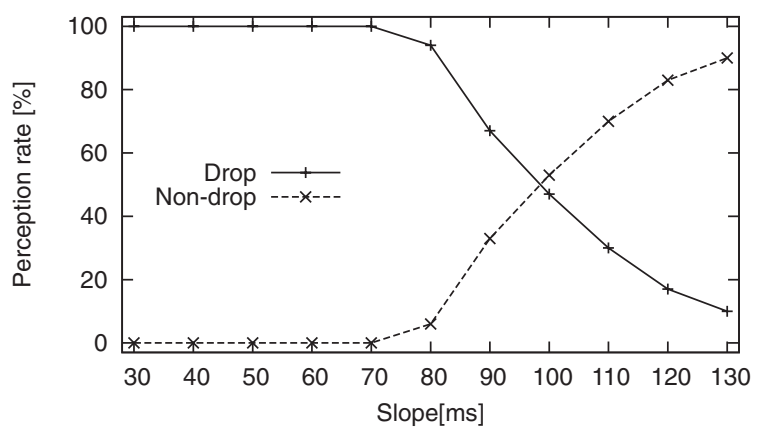

Fig. 11 Perceptions of shifted $F_{0}$ patterns of drop tone.

spectrum. The sounds were combined into a pattern of "/ma/ test-sound /ma/" in which /ma/ was the analysis-synthesis sound with a level tone and the connecting interval was $70 \mathrm{~ms}$. The test syllable length was adjusted to the longest length (i.e., $130 \mathrm{~ms}$ slope) by inserting silence. The listeners were Sam and Luong.

b) Results

The results of listening tests are shown in Fig. 11. The perception rate is satisfactorily high with an $80 \mathrm{~ms}$ slope, and becomes higher with further shift of the reference slope to the left. This result is consistent with the result for multislope $F_{0}$ patterns.

\subsubsection{Categorical test}

\section{a) Procedure}

Categorical tests were performed for shifted $F_{0}$ patterns of the drop tone. A pair of $30 \mathrm{~ms}$ neighbor sounds was played once with a short pause between them. There were 10 pairs and each pair of sounds was repeated 10 times randomly. The listener was asked to decide whether the two sounds are the same tone or not. Each syllable was tested separately. The listeners were Sam and Luong.

b) Results

The results of listening tests are shown in Fig. 12. The peak values of the differences are from " $80 \sim 110$ " to "100 130" for Sam and from "80 110" to "90 120" for Luong. There are some categorical values for separated syllables and for each listener. However, there is no common threshold for the average result of syllables and the two listeners.

\section{FINAL PART OF BROKEN TONE}

\subsection{Procedure}

We synthesized the broken tone with the following speech parameters: average $F_{0}$ of the broken tone, the spectrum of the level tone, and the power of the level tone with V-shape power control. For the power pattern, we selected the value (5-frame; weighting factor 0.875 ) from the results in Section 3. For the $F_{0}$ pattern, the starting point of the final part was determined manually to be around the middle of the vowel with reference to its 


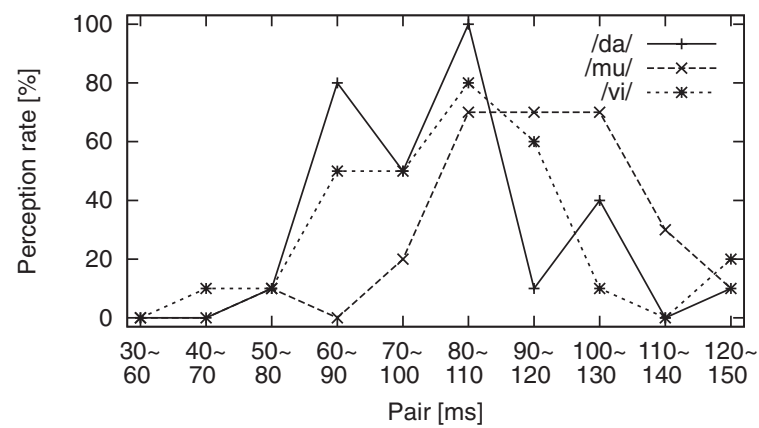

a) Listener: Sam

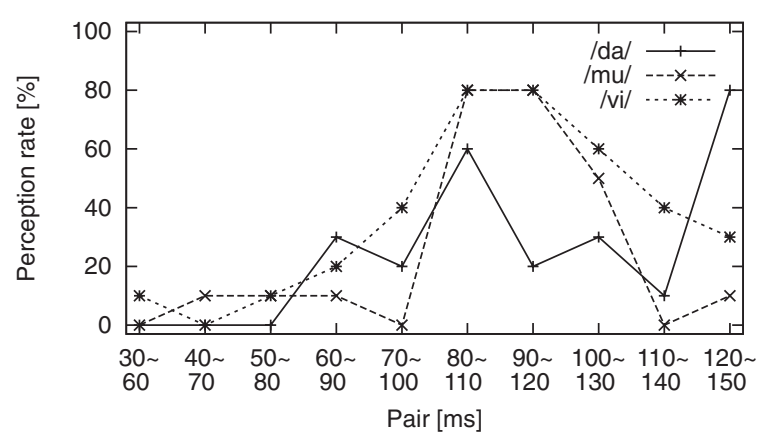

b) Listener: Luong

Fig. 12 Categorical test results for shifted $F_{0}$ patterns of drop tone.

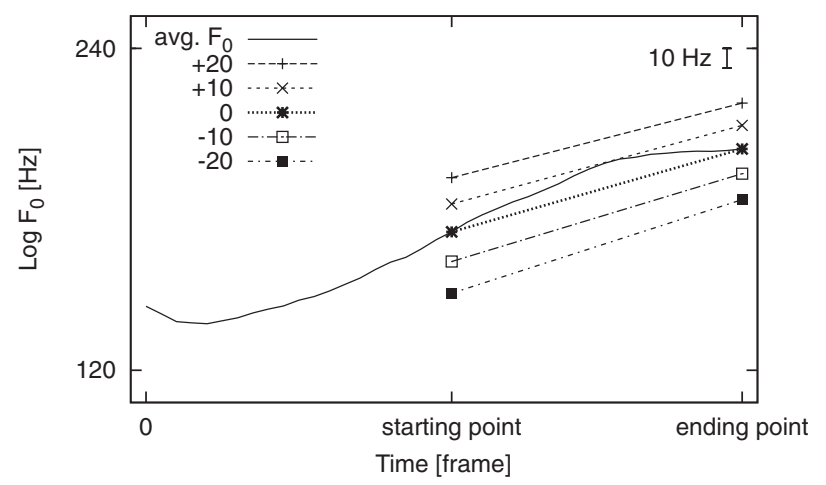

Fig. 13 Shifted $F_{0}$ patterns of broken tone.

spectrum, while the ending point was the end of the syllable. We linearized $F_{0}$ of the final part of the broken tone from the broken tone average $F_{0}$ contour. This reference line of $F_{0}$ is denoted as " $0 \mathrm{~Hz}$." We change $F_{0}$ of the final part of the broken tone with two kinds of patterns.

- Shifted $F_{0}$ patterns (Fig. 13): shift $+20,+10,0,-10$, and $-20 \mathrm{~Hz}$

- Multislope $F_{0}$ patterns (Fig. 14): the starting point of the final part is retained and only their ending points are changed as $+20,+10,0,-10,-20 \mathrm{~Hz}$ and flat (i.e., the value of the ending point is equal to that of the starting point).

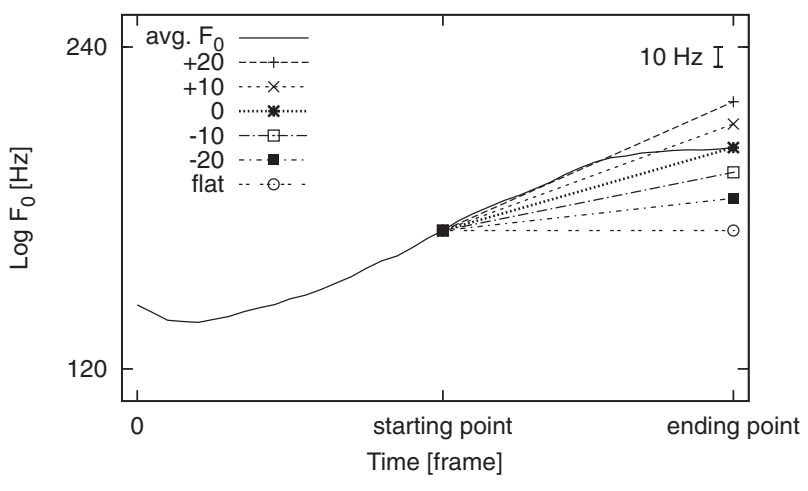

Fig. 14 Multislope $F_{0}$ patterns of broken tone.

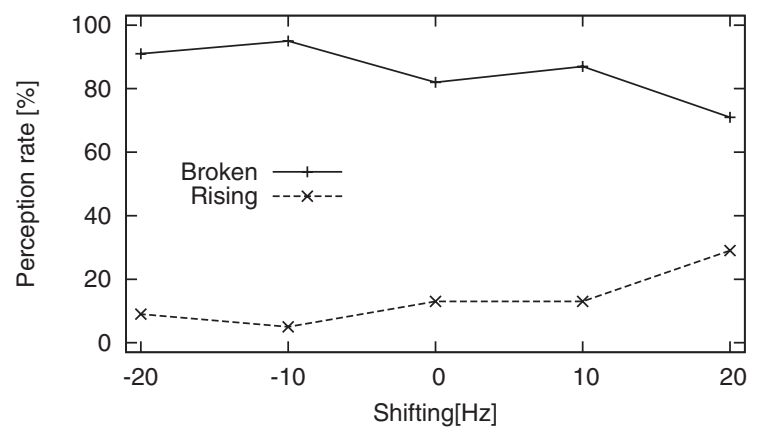

Fig. 15 Perceptions of shifted $F_{0}$ patterns of broken tone.

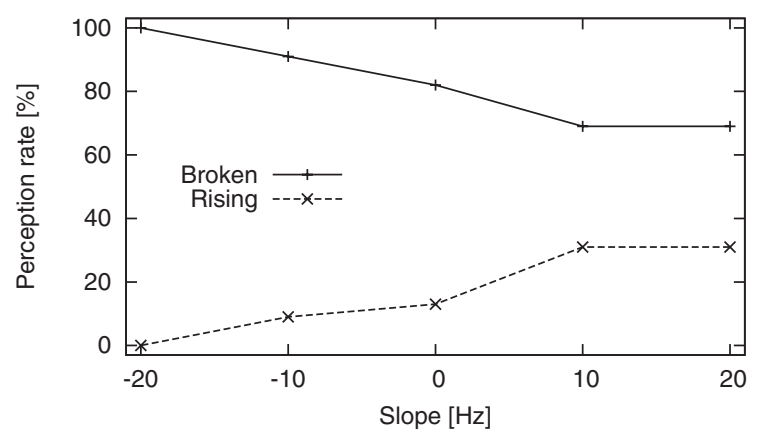

Fig. 16 Perceptions of multislope $F_{0}$ patterns of broken tone.

In the listening test, the sounds were prepared with a balance of six tones. The isolated sounds were mixed together and repeated five times randomly. The listeners were Sam, Loan (female), and Ha (female). Loan and $\mathrm{Ha}$ are also from Northern Vietnam, have normal hearing abilities, and were 28 and 26 years old, respectively.

\subsection{Results}

The results are shown in Figs. 15 and 16. The perception rates as the broken tone are high, but there are no clear thresholds for either shifted $F_{0}$ patterns or multislope $F_{0}$ patterns. These experimental results imply 
that the final part of the broken tone has only a minor effect on the perception of the broken tone. For the flat pattern, the average rate for perception as a level tone is $42 \%$. This means that the final part of the broken tone requires a rising $F_{0}$ pattern.

For power control, the results in Section 3 show that the perception rates of the broken tone were high with weighting factors less than or equal to 0.875 . We selected the (5-frame; weighting factor 0.875 ) pattern because it was close to the $50 \%$ perception point. The results in Section 3 and of this experiment suggest that i) with smaller weighting factors, the perception as a broken tone is high, and there is no clear threshold between broken and rising tones; and ii) the greater the weighting factor is, the more the sound is perceived as a rising tone. We believe that even if other values of the weighting factor are used, there will be no change in our conclusion.

\section{CONCLUSIONS}

We studied the perceptual characteristics of Vietnamese broken and drop tones, wherein glottal features exist. The broken tone has a glottal constriction in the middle, while the drop tone has a glottal stop at the end. We considered the effects of the power and $F_{0}$ on the perception of these tones.

Four experiments were conducted. In the first experiment, to reconfirm the effectiveness of power control for the broken tone, the listening tests were carried out under difficult test conditions. The power dip pattern resulted in high intelligibility of the synthetic sound of the broken tone, and a reasonable weighting factor is 0.875 . In the second experiment, we examined the similarity between the first half of the broken tone and the drop tone. We found that the drop tone and the first half of the broken tone are comparable, i.e., the first half of the broken tone can be replaced by the drop tone with very high perception rates, and conversely, the drop tone can be replaced by the first of the broken with reasonable rates. In the third experiment, for the final part of the drop tone, we found that the perceptual threshold of $F_{0}$ is around $80 \mathrm{~ms}$ for the glottal stop. In the fourth experiment, for the final part of the broken tone, the results showed that $F_{0}$ has a minor effect on the perception.

For the pair of broken and drop tones, the similarity at the beginning parts (i.e., exchangeable) and the contrast at their end parts (i.e., the drop tone ends with a glottal stop, but the broken tone has a final part) can give a better understanding of Vietnamese linguistics. In future work, it may be interesting to study the similarity between glottal features in several languages and in Vietnamese.

\section{ACKNOWLEDGMENTS}

The authors thank the native Vietnamese listeners (Sam, Luong, Loan, Ha, and Phuong) who participated in the listening tests in the evaluations.

\section{REFERENCES}

[1] T. T. Doan, Vietnamese Phonetics (Hanoi National University Publishing, Hanoi, 1999), pp. 100-111 (in Vietnamese).

[2] M. Shimizu and M. Dantsuji, "A new proposal of laryngeal features for the tonal system of Vietnamese," Proc. ICSLP 2000, Vol. 2, pp. 519-522 (2000).

[3] D. Crystal, A Dictionary of Linguistics and Phonetics, 6th ed. (Blackwell Publishing Ltd., Oxford, 2008), p. 486.

[4] Z. Bao, The Structure of Tone (Oxford University Press, New York, 1999), pp. 100-111.

[5] M. Yip, Tone (Cambridge University Press, Cambridge, 2002), pp. 1-10.

[6] H. Mixdorff, N. Bach, H. Fujisaki and L. Mai, "Quantitative analysis and synthesis of syllabic tones in Vietnamese," Proc. Eurospeech 2003, pp. 177-180 (2003).

[7] T. T. Do and T. Takara, "Vietnamese text-to-speech system with precise tone generation," Acoust. Sci. \& Tech., 25, 347353 (2004).

[8] International Phonetic Association, Handbook of the International Phonetic Association: A Guide to the Use of the International Phonetic Alphabet (Cambridge University Press, Cambridge, 1999), p. ix.

[9] S. Imai, "Log magnitude approximation (LMA) filter," Trans. IECE Jpn., J63-A, 886-893 (1980) (in Japanese).

[10] T. T. Do and T. Takara, "Multi-platform visual interactive speech analysis-synthesis system," Proc. ICEE 2008, pp. O.161.1-O.161.4 (2008).

[11] T. Takara, "Experimental study on perception of the glottal explosive of the Japanese Ryukyu dialect," Proc. EuroSpeech'95, pp. 953-956 (1995).

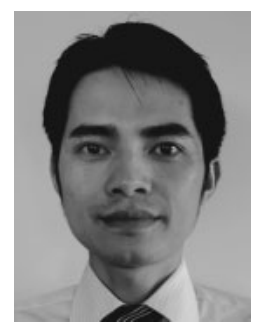

Tu Trong Do received his B.E. degree in Electronics and Telecommunications from Hanoi University of Technology, Vietnam, in 1999 and M.E. degree in Information Engineering from University of the Ryukyus, Japan, in 2002. He has studied in the doctoral program of the Department of Information Engineering, University of the Ryukyus, Japan. His research interests are speech processing and its interactive applications.

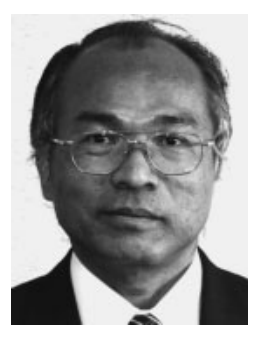

Tomio Takara received his B.S. degree in Physics from Kagoshima University, Japan, in 1976 and M.E. and Dr.Eng. degrees in Information Processing from Tokyo Institute of Technology, Japan, in 1979 and 1983, respectively. During 1991-1992, he studied at Carnegie Mellon University as a visiting scientist. He has been a Professor in the Department of Information Engineering, University of the Ryukyus, Japan, since 1995. He is the recipient of the 1990 Okinawa Society Award for Encouragement of Study on Okinawa. $\mathrm{He}$ is presently interested in spoken language processing and machine intelligence. 